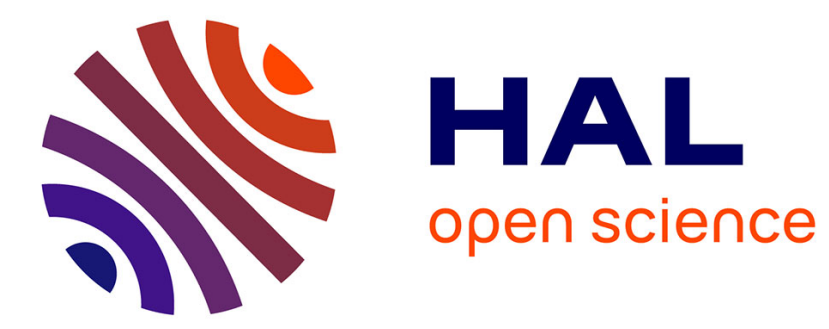

\title{
QUALITATIVE ANALYSIS OF THE DYNAMIC CRACK INITIATION AND PROPAGATION
}

\author{
M. Watanabe
}

\section{To cite this version:}

M. Watanabe. QUALITATIVE ANALYSIS OF THE DYNAMIC CRACK INITIATION AND

PROPAGATION. Journal de Physique IV Proceedings, 1991, 01 (C3), pp.C3-713-C3-718. 10.1051/jp4:19913100 . jpa-00249902

\section{HAL Id: jpa-00249902 https://hal.science/jpa-00249902}

Submitted on 1 Jan 1991

HAL is a multi-disciplinary open access archive for the deposit and dissemination of scientific research documents, whether they are published or not. The documents may come from teaching and research institutions in France or abroad, or from public or private research centers.
L'archive ouverte pluridisciplinaire HAL, est destinée au dépôt et à la diffusion de documents scientifiques de niveau recherche, publiés ou non, émanant des établissements d'enseignement et de recherche français ou étrangers, des laboratoires publics ou privés. 


\title{
QUALITATIVE ANALYSIS OF THE DYNAMIC CRACK INITIATION AND PROPAGATION
}

\author{
M. WATANABE \\ Kinki University, Faculty of Engineering, Umenobe, Takaya, \\ Higashi-Hiroshima 729-17, Japan
}

résumé: Des résultats expérimentaux de fissuration rapide sont analysés à partire de l'équation de conservation de l'energie prenant en compte l'effet de microcavités générées au sommet de la fissure. Le rayon moyen des microcavités est supposé crô̂tre selon une loi de puissance $\mathfrak{t}^{\alpha}$ et le volume des microcavitésest défini par $\mathrm{r}^{\mathrm{D}}$ et leur densité de distribution $\operatorname{par}^{-\beta}$. les valeurs de $\alpha$ sont estimées en fonction de celles de $\beta$. La rugosité de surface peut être analysée qualitativement à partir de la détermination expérimentale des paramètres $D$ et $\beta$. La condition postulée d'incitation de la fissure est étendue à la phase de propagation. Les résultats sont en accord qualitatif avec les expériences.

Abstract-Experimental results of the fast fracture of the crack is analyzed based on the extended energy balance equation in which the effect of micr ovoids generated at the tip of the crack is taken into account. We assume that the average radius $r$ of microvoids grow in time as $r \propto t^{\alpha}$, i.e., micr ovoids grow in the self-similar manner, statistically. It's volume is defined as $\mathrm{r}^{\mathrm{D}}$ and the density distribution function $\mathrm{n}(\mathrm{r})$ of the microvoids is assumed to be $n \propto r^{-\beta}$, where $D$ and $\beta$ are numbers. The surface roughness $\langle\mathrm{r}\rangle$ defined as the average radius of microvoids is compared with the experiment. Thus we can estimate the numbers $\alpha$ and $\beta$ as $\alpha \fallingdotseq 5.5$ for 1$\rangle$ $\beta>0$ and $\alpha>5.5$ for $2>\beta>1$, respectively. We have shown that the surface roughness which is called as "mirror", "mist" and "hackle" can be qualitatively analyzed from the energy balance equation if the parameters $D$ and $\beta$ are measured experimentally. Furthermore, we have postulated the initiation condition of the dynamically loaded crack and extended it to the propagation stage. This postulate and it's extension show the qualitative agreement $w$ ith the experim ental observations.

\section{Introduction.}

We analyze the results of the series of the experiments on the fast fr actute perfor med by Ravi-Chandar and Knauss /1/. The specimen material used in their experiment is Homalite 100 and it's size is chosen such that the reflected waves do not interact with the crack tip for the duration of the experiment and thus a literally unbounded (plate) medium is simulated. They applied the tensile stress using the electromagnetic loading device and measured the stress intensity factor history by the method of caustics. Investigating various different aspects of the dynamic fracture, they found that crack propagation occurs by the linking up of many microvoids(or microcracks). Our analysis is based on this observation and the assumption that microvoids grow in the self-similar manner statistically. 


\section{II.T he grow th law and the density of the microvoids.}

The micromechanics of $r$ upture of polymers under tensile stress was thoroughly investigated $/ 2 \%$. For example, physical mechanism of the generation of microvo ids is clar if ied in the frame work of ther mal activation theory of fracture of solids. $Z$ hurkov and $\mathrm{Kuksenko} / 3 /$ propposed the hypothesis that the formation and development of micr ovoids (or sub microcr acks) play a dominant role in the micromechanics of fracture of polymers. They measur ed the microvoid distribution in the direction of the crack grow th as show $\mathrm{n}$ in Fig. 1 by the small angle $\mathrm{X}$-ray scattering. They also compar ed the scattering curves during the initial stage of fracture long before rupture, to scattering before rupture and found the signif icant changes in the sizes of microvoids. The enelarged microvoids are $\mathrm{r}$ egarded as the coalescence of the primary microvoids.

In the experiment of the dynamic fracture, Ravi-Chander and Knauss found that crack propagation occurs by the linking up of many microvoids(or microcracks). The number of growing microvoids that are activated is a function of the stress intensity factor and the distr ibution of voids in the material itself. The fractured surface depth $d$ is measured along the crack path and it's variation is shown as a function of the measured stress intensity factor. They established a quan titative correlation betw een the stress intensity factor and the surface roughness as show $\mathrm{n}$ in Fig. 2. We find the following relation from Fig. 2

$$
\frac{\mathrm{d}}{\mathrm{d}_{\max }}=\left(\frac{\mathrm{K}_{\mathrm{I}}}{\mathrm{K}_{0}}\right)^{11.1}
$$

wher $e K_{1}$ is the stress intensity factor and $K_{0}$ is a constant.
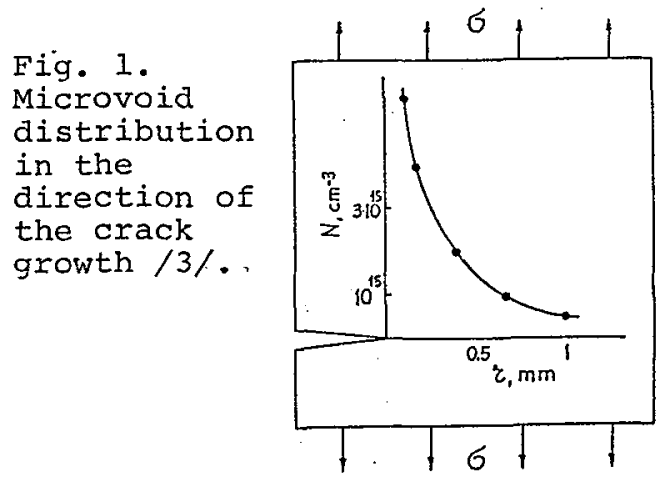

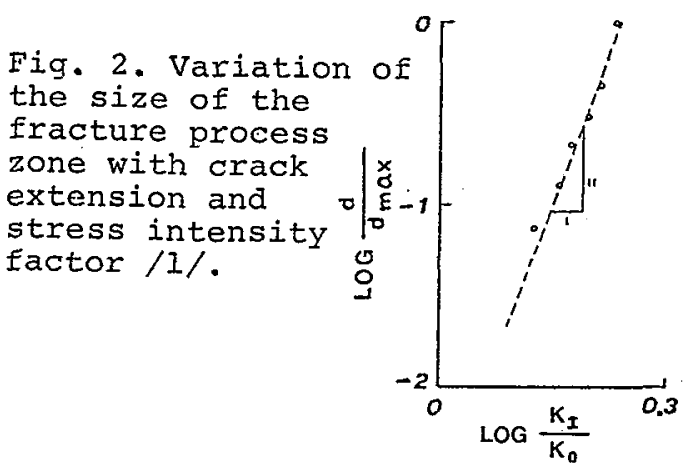

The diameters of the microvoids measured in the static case range from 0.009 to 0.3 $\mu \mathrm{m}$ for different polymers $/ 3 \%$. When the applied stress is sufficien tly small, the radius ro of the microvoid is determined by the thermal equilibrium condtion. In the dynamic case, the size of the microvoids incr eases by various different mechanisms as the stress intensity factor increases. Finally the microvoid grow: to be a small micr ocrack whose leng th $r$ eaches to the range of milimeter. We assume that the character istic length of the micr ovoids does not exist in these grow th processes. In such situations, the microvoids grow in the self-similar manner statistically $/ 4 /$ and the radius $r$ of the microvoids can be assumed as

$$
r=r_{0}\left(\frac{t}{t_{0}}\right)^{\alpha} \text {, }
$$

wher $e$ to is a char acteristic time for generation of microvoids in thermal equilibrium. The growth rate of the microvoid is determined by the number $\alpha$. As the crack propagates, the microvoids near the tip of the crack is activated. Suppose that the microvoids start to grow when the distance between the crack and the microvoids becomes $R$. In this case, the following relation holds

$$
\frac{K_{I}}{\sqrt{2 \pi R}}=\sigma_{c},
$$


where the quantity. $\sigma_{c}$ is the average critical stress for the growth of the microvoids. Since the crack propagates by the constant velocity $\hat{\alpha} / 1 /$, the interaction time $\tau_{\text {int }}$ betw een the crack and the microvo ids can be obtained by

$$
\tau_{\text {int }}=\frac{R}{a}=\frac{K_{I}^{2}}{2 \pi \sigma_{c}^{2}} \dot{a}
$$

The maximum radius $r_{m}$ of the micr ovoids in the fractured surface can be expr essed as

$$
\mathrm{r}_{\mathrm{m}}=\mathrm{r}_{0}\left(\frac{\mathrm{R}}{\dot{a} t_{0}}\right)^{\alpha}=\mathrm{r}_{0}\left(\frac{\mathrm{K}_{\mathrm{I}}^{2}}{2 \pi \sigma_{\mathrm{c}}^{2} \dot{a} t_{0}}\right)^{\alpha}
$$

The interaction time $\tau_{\text {int }}$ betw een the crack and the microvoids changes due to the shielding effect associated with the inhomogenuity of the specimen and other reasons which is not discussed here. Thus the microvoids with various different radius are produced. We assume the density distribution function of the microvoids as

$$
\mathrm{n}=\mathrm{n}_{0}\left(\mathrm{r}_{0} / \mathrm{r}\right)^{\beta}
$$

wher $\mathrm{e} \mathrm{n}_{0}$ is the density of microvoids in ther mal equilibr ium.

The average radius $\langle\mathrm{r}\rangle$ of the micr ovoids in the fractured surface can be defined as

$$
\langle r\rangle=\int_{r_{0}}^{r_{m}} r n(r) d r / \int_{r_{0}}^{r_{m}} n(r) d r
$$

The aver age radius $\langle\mathrm{r}\rangle$ of the microvoids is regarded as the quantity which is proportional to the surface roughness $d / d_{\max }$ defined by $E q$. (1), The $K_{I}$ dependence of the aver age radius $\langle r\rangle$ can be found in the following

$$
\begin{aligned}
& \langle r\rangle=\frac{1-\beta}{2-\beta} r_{m}=\frac{1-\beta}{2-\beta} r_{0}\left(\frac{K_{I}^{2}}{2 \pi \sigma_{C}^{2} \dot{a} t_{0}}\right)^{\alpha} \\
& \alpha \mathrm{K}_{1}^{11.5} \quad \therefore \alpha \approx 5.5 \\
& \left.\left.\langle\mathrm{r}\rangle=\frac{\beta-1}{2-\beta} \mathrm{r}_{0}\left(\frac{\mathrm{T}_{m}}{\mathrm{r}_{0}}\right)^{2-\beta}=\frac{\beta-1}{2-\beta} \mathrm{r}_{0}\left(\frac{\mathrm{K}_{\mathrm{I}}^{2}}{2 \pi \sigma_{C}^{2} \dot{a} \mathrm{t}_{0}}\right)^{\alpha(2-\beta)} \quad \text { for } 2\right\rangle \beta\right\rangle 1 \\
& \alpha \mathrm{K}_{\mathrm{I}}^{11.5} \quad \therefore \alpha(2-\beta) \approx 5.5 \\
& \text { for } 1>\beta>0
\end{aligned}
$$

wher $\mathrm{e}_{0} / \mathrm{r}_{\mathrm{m}}\langle\langle 1$ is used. In the latter case, we find $\alpha>5.5$ from the condition 2$\rangle \beta\rangle$ 1. If $\beta=1$ or $\beta=2$, the average radius $\langle\mathrm{r}\rangle$ depends on $\ln \left(\mathrm{r}_{\mathrm{m}} / \mathrm{r}_{0}\right)$, which is not observed in the exper iment. In the case $\beta\rangle 2$, we find $\langle\mathrm{r}\rangle=(\beta-1) \mathrm{r}_{0} /(\beta-1)$ which is independent of $K_{1}$. Thus we have disregarded these cases. The average density $\langle\mathrm{n}\rangle$ defined by

$$
\langle\mathrm{n}\rangle=\int_{\mathrm{r}_{0}}^{\mathrm{x}_{\mathrm{m}}} \mathrm{n}(\mathrm{r}) \mathrm{dr} / \int_{\mathrm{r}_{0}}^{\mathrm{T}_{\mathrm{m}}} \mathrm{dr}
$$

plays an important role in considering the behavior of the microvoids. In $\mathrm{D}$ dimensional space the aver age distance between microvoids is given by $\langle n\rangle^{-1 / D}$. When the average radius $\langle r\rangle$ of the microvoids reaches the distance $\langle n\rangle^{-1 / D}$, microvoids coalesce $w$ ith each other. In Table 1 , we show the values of the quantities $\langle\mathrm{n}\rangle / \mathrm{n}_{0}$ and $\langle\mathbf{n}\rangle\langle\mathrm{r}\rangle^{\mathrm{D}} /\left(\mathrm{n}_{0} \mathrm{P}\right)$

$$
\begin{aligned}
& \text { B) } 2 \quad \beta=2 \\
& \text { 2) } \beta>1 \\
& \beta=1 \\
& 1>\beta>0 \\
& \langle\mathrm{n}\rangle / \mathrm{n}_{0} \\
& \frac{r_{0}}{(\beta-1) r_{m}} \\
& \frac{r_{0}}{r_{m}} \quad \frac{r_{0}}{(\beta-1) r_{m}} \\
& \frac{r_{0}}{r_{m}} \ln \left(\frac{r_{m}}{r_{0}}\right) \\
& \frac{1}{1-\beta}\left(\frac{\mathrm{T}_{0}}{\mathrm{I}_{\mathrm{m}}}\right)^{\beta} \\
& \frac{\langle n\rangle\langle r\rangle^{D}}{n_{0} r_{0}^{D}} \quad \frac{(\beta-1)^{D} r_{0}}{(\beta-2)^{D} r_{m}} \quad \frac{r_{0}}{r_{m}}\left(\ln \frac{r_{m}}{r_{0}}\right)^{D} \\
& \frac{(\beta-1)^{D-1}}{(2-\beta)^{D}}\left(\frac{T_{0}}{r_{m}}\right) D(\beta-2)+1 \\
& \left(\frac{\frac{r_{m}}{r_{0}}}{\ln \frac{r_{m}}{r_{o}}}\right)^{D-1} \\
& \frac{(1-\beta)^{D-1}}{(2-\beta)^{D}}\left(\frac{r_{m}}{r_{0}}\right)^{D-\beta}
\end{aligned}
$$


We find from Table 1 that the average number $\langle n\rangle$ of the microvoid decreases as the microvoids grow due to the coalescence among them. The value of $\langle n\rangle\langle r\rangle^{D}$ decr eases for $\beta \geqq 2$, while it could increase if $\beta<2$. These conditions are closely related with the branching and $w$ ill be discussed in the next section.

III. The dy namic crack propagation and the branching.

When the stress intensity factor is not so large in compared with $K_{I C}$ and the crack velocity is relatively small, say $=240 \mathrm{~m} / \mathrm{s}$, the energy balance equation of Griffith can be applied.

$$
\left(1-\frac{\dot{a}}{c_{R}}\right) G^{*}=2 \gamma_{0}
$$

wher e $c_{R}$ is the velocity of the Rayleigh wave and $G^{*}$ the "static" energy release rate. In this case the quasi-static condition is satisfied and the effect of the term ( ) in L.H.S. of Eq. (11) is observed $/ 1 /, 15 \%$. The fractured surface is smooth and is characterized as the "mir ror" state. As the stress intensity factor increases the fractured surface changes from "mirror". to the "mist" or "hackle" state. In this case, the additional ener gy which is absorbed by the microvoids should be added in Eq. (II),

$$
\left(1-\frac{\dot{a}}{c_{R}}\right) G^{*} w d a=2 \gamma_{0} w d a+\gamma_{0} \sum_{i} \Delta S_{i}
$$

The quantity $\Delta S_{i}$ repr esents the increased area of the microvoid during the period of $t$ and $t+d t . \Delta S_{i}$ can be written as $\Delta S_{i}=c_{i}\left(D_{i}-1\right) D_{i}^{D_{i}-2} d r_{i}$, where $r_{i}$ and $D_{i}$ are the radius and the spacial dimension of the $\mathrm{i}$-th microvoid, respectively. The quantity $w$ is the width of the specimen. Making use of $\mathrm{Eq}$. (2), we find

$$
\mathrm{dr}=\frac{\alpha r_{0}}{\mathrm{t}_{0}}\left(\frac{\mathrm{r}}{\mathrm{T}_{0}}\right){ }^{1-(1 / \alpha)} \mathrm{dt}
$$

Eq. (13) is used in calculating the averaged quantities of $\Delta S_{i}$. The microvoid is generated in the small volume $\Delta V=w R\langle I\rangle$, and the average number of the microvoids can be written as $\langle n\rangle \Delta V$. Thus the second term in R.H.S. of Eq. (12) can be written as

$$
\gamma_{0} \sum_{i} \Delta S_{i}=\gamma_{0}\langle n\rangle \Delta V \frac{\alpha r_{0}^{1 / \alpha}}{t_{0}}(D-1) c\left\langle r^{D-1-(1 / \alpha)}\right\rangle d t
$$

where $c$ is a constant and $D$ the average spacial dimension of the microvoids. In calculating the quantity $\left\langle\mathrm{r}^{\mathrm{D}-1-(1 / \alpha)}\right\rangle$, we have to divide it into numerous different cases. If the condition $1>\beta>0$ is satisfied, Eq. (12) can be written as

$$
\begin{aligned}
& \left(1-\frac{\dot{a}}{C_{R}}\right) G^{*}-2 \gamma_{0} \\
= & \gamma_{0} \frac{\alpha^{2}(1-\beta)(D-1) c}{(2-\beta)\{\alpha(D-\beta)-1\}} n_{0} r_{0}^{D}\left(\frac{\tau_{\text {int }}}{t_{0}}\right)^{\alpha(D-\beta)} \\
= & \gamma_{0} \frac{(2-\beta)^{D-1} \alpha^{2}(D-1) c}{(1-\beta)^{D-2}\{\alpha(D-\beta)-1\}}\langle n\rangle\langle r\rangle^{D}
\end{aligned}
$$

Substituting the numbers $\alpha=5.5$ and $\beta=0.7$, we find

$$
\text { R.H.S. of Eq. (15) } /\langle n\rangle\langle r\rangle^{D} \approx 630 \gamma_{0} \text { for } D=3, c=4 \pi \text { and } \approx 40 \gamma_{0} \text { for } D=2, c=2 \pi \text { s }
$$

The condition $\langle\mathrm{n}\rangle\langle\mathrm{r}\rangle^{3} \approx 1$ yields $\tau_{\text {int }} / \mathrm{t}_{0} \approx\left(25 / \mathrm{n}_{0} \mathrm{r}_{0}^{3}\right)^{1 / 13}$ for the former and $\tau_{\text {int }} / t_{0} \approx\left(5.6 / n_{0} I_{0}^{2}\right)^{1 / 7.2}$ for the latter case. Thus the surface roughness can be qualitatively analyzed from the ener gy balance equation if one measures the parameters such as $D$ and $\beta$ besides the measurement show $n$ in Fig. 2. Since the numerical factor in R.H.S. of Eq. (15) is $r$ ather lar ge in compar ed with $2 \gamma_{0}$, the branching condition will be given by the numerical value of $\langle n\rangle\langle r\rangle$ and it probably lies within the inequqlitities 10 $>\tau_{\text {int }} y_{t_{0}}>1$ This condition can be expressed by the stress intensity factor using Eq. (4). Since the condition $\langle\mathrm{n}\rangle\langle r\rangle^{\mathrm{D}} \approx 1$ will not be satisfied for $\beta \geqq 2$ provided $\mathrm{n}_{0} \mathrm{r}^{\mathrm{P}}\langle 1$, we disr egard these cases. The energy balance equation for $2>\beta>1$ is 


$$
\left.\left\{\left(1-\frac{\dot{a}}{c_{R}}\right) G^{*}-2 \gamma_{0}\right\}=\gamma_{0} \frac{\alpha^{2} c(D-1)(2-\beta)^{D-1}\langle n\rangle\langle r\rangle^{D}}{(\beta-1)^{D-2}\{\alpha(D-\beta)-1\}}\left(\frac{\tau_{\text {int }}}{t_{0}}\right)^{\alpha(\beta-1)(D-2)} \text { if } \alpha(D-\beta)\right\rangle 1
$$

Substituting the numbers $\alpha=7$ and $\beta=1.3$ as an example we find $\left\{\left(1-\frac{\dot{a}}{c_{R}}\right) G^{*}-2 \gamma_{0}\right\} /\langle n\rangle\langle r\rangle^{D} \approx 180 \gamma_{0}\left(\frac{\tau_{i n t}}{t_{0}}\right)^{2.1}$ for $D=3, c=4 \pi$ and $\approx 55 \gamma_{0}$ for $D=2, c=2 \pi$ The condition $\langle n\rangle\langle r\rangle^{D} \approx 1$ yields $\tau_{\text {int }} / t_{0} \approx\left(4 / \mathrm{n}_{0} \mathrm{r}_{0}^{3}\right)^{1 / 7.7}$ for the former and $\tau_{\text {int }} / t_{0} \approx\left(2 / n_{0} r_{0}^{2}\right)^{1 / 2.8}$ for the latter case. These numerical examples lead to the similar conclusion to the case $1>\beta>0$.

IV. The dy namic cr ack initiation.

Ravi-Chander and Knauss / // found the time dependence of the critical stress intensity factor $\mathrm{K}_{\mathrm{IC}}$ for crack initiation as show $n$ in Fig. 3. In the quasi-static condition, the crack start to propagate if the condition $\mathrm{K}_{\mathrm{IC}}=\mathrm{K}_{\mathrm{C}}$ is satisfied. This condition is equivalent ${ }_{*}$ to the energy balance equation $G=2 \gamma_{0} . T$. The experimental observation $K_{I C} \propto t^{-2}$ for $t$ $\leq 50 \mu s$ indicates that the additional condition for the dynamic crack initiation is required, which is different from the quasistatic ener gy balance equation. In order to explain the dy namic crack initiation shown in Fig. 3, we postulate the following condition for the crack initiation. $\mathrm{S}=\mathrm{S}_{\mathrm{C}}$

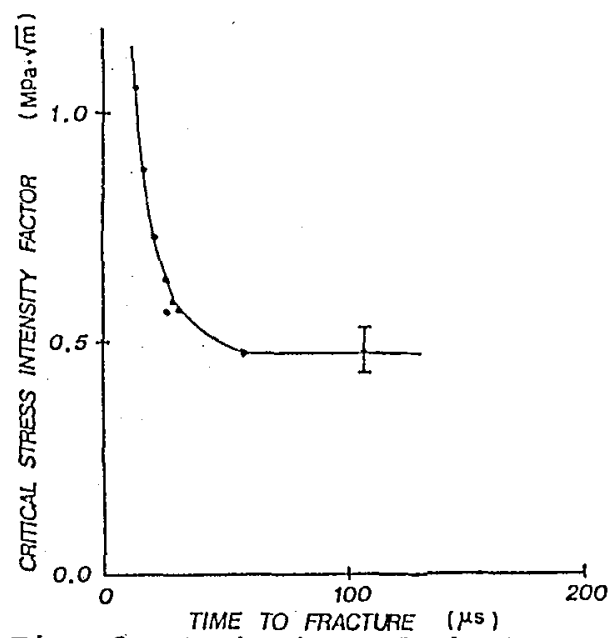

Fig. 3. Variation of the stress intensity factor required for initiation with time to fracture/1/ where $S$ is the surface area fractured at the tip of the crack and $S_{C}$ is the critical surface area for the crack initiation. The surface area $S$ increases in time and it's time dependence is assumed to be

$$
S(t)=S_{1}\left(\frac{t}{t_{1}}\right)^{\alpha_{s}} \quad \text { for } t \leq t_{1}
$$

where $S_{1}=w R_{I C}$ and $t_{1}=50 \mu \mathrm{s}$ from Fig., 3 and $\alpha_{S}$ is the number. The quantities $R_{\mathrm{IC}}$ and $\mathrm{R}_{\mathrm{C}}=\mathrm{S}_{\mathrm{C}} / \mathrm{w}$ are defined as

$$
\frac{\mathrm{K}_{\mathrm{IC}}}{\sqrt{2 \pi \mathrm{R}_{\mathrm{IC}}}}=\sigma_{\mathrm{C}}, \quad \frac{\mathrm{K}_{\mathrm{C}}}{\sqrt{2 \pi \mathrm{R}_{\mathrm{C}}}}=\sigma_{\mathrm{C}} \text {. }
$$

Suppose that the crack start to grow with $t<50 \mu \mathrm{s}$. In this case we find the following equation

$$
\mathrm{S}_{1}\left(\frac{\mathrm{t}}{\mathrm{t}_{1}}\right)^{\alpha_{s}}=\mathrm{w} \mathrm{R}_{\mathrm{C}} \quad \therefore \quad \mathrm{R}_{\mathrm{IC}}=\mathrm{R}_{\mathrm{C}}\left(\frac{\mathrm{t}_{1}}{\mathrm{t}}\right)^{\alpha_{s}} .
$$

Eq. (21) can be written as

$$
K_{\text {IC }}^{2}=K_{C}^{2}\left(\frac{t_{1}}{t}\right)^{\alpha_{s}} \text {, }
$$

which determines the effective surface energy $2 \gamma(\mathrm{t})$ such that the energy balance equation $G^{k}=2 \gamma(t)$ holds. Comparing Eq. (22) with Fig. 3, we find $\alpha_{S}=4$. Note here that the quasi-static initiation of the crack is included in Eq. (22) if one sets $t=t_{1}$ and $\gamma=\gamma_{0}$. in the ener gy balance equation.

Fig. 4 shows the stress intensity factor and the crack extension histor $y / 1 /$. They observe that the arrival of the wave reflected from the boundaries at about $150 \cdot \mu \mathrm{s}$ causes the stress intensity factor to increase as in Fig. 4 (solid line) and the crack velocity changes from $240 \mathrm{~m} / \mathrm{s}$ to $350 \mathrm{~m} / \mathrm{s}$. They conclude from these observations that 
crack travling at a low velocities, typically below $300 \mathrm{~m} / \mathrm{s}$, can change their velocity of propagation upon encountering str ess waves.

According to the postulate show $n$ in Eqs. (18) and (19), the crack starts to propagate at $S=S_{C}$ and the crack velocity is determined by the following equation

$$
\dot{a}=\frac{1}{w}\left(\frac{d S}{d t}\right)_{S=S_{c}}=\left(\frac{\alpha_{S}}{t}+\frac{2 d K_{I C}}{K_{I C} d t}\right) S_{C}
$$

The crack velocity is determined by the initiation condition of the crack which consist of the two terms of ( ) in R.H.S. of Eq. (23). The former show $s$ the characteristic time of the grow th of $S(t)$ , while the latter is associated with the dynamic behavior of the stress intensity factor at the initiation. Once the crack starts to propagate, we do not have to restrict these two terms at the initiation. Thus we can replace $\mathrm{K}_{\mathrm{IC}}(\mathrm{t})$ by $\mathrm{K}_{\mathrm{I}}(\mathrm{t})$ in Eq. (23). Numerical evaluation of the two terms in R.H.S. of Eq. (23) indicates that the first ter $m$ is always larger than the second term except at encountering the stress wave. When the crack encounters with the stress wave at $\mathrm{t}=150 \mu \mathrm{s}$ (solid line of Fig. 4), we find

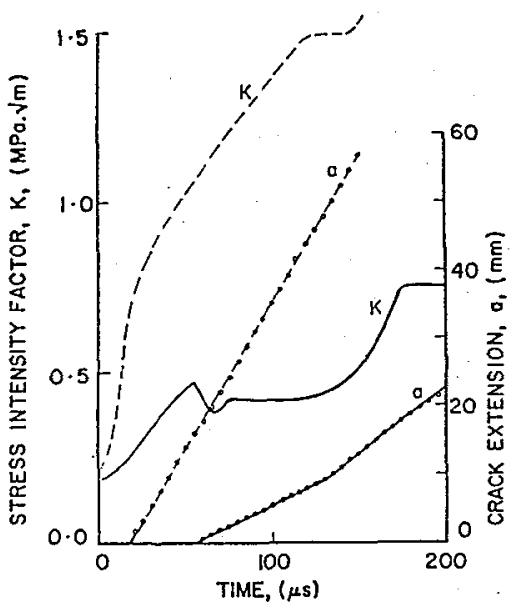

Fig. 4 Stress intensity factor and crack extension history/1/

$$
\frac{1}{\mathrm{~K}_{\mathrm{I}}} \frac{\mathrm{dK}}{\mathrm{dt}} \geq \frac{\alpha_{\mathrm{S}}}{\mathrm{t}}
$$

When the crack encounters with the stress wave, the experimental observation indicates that the characteristic time of the grow th of $S(t)$ changes and remains unchanged there after. In order to clarify the theoretical $r$ easons for it, one has to look into the the atom ic motion of the mater ial which is beyond the scope of this work.

\section{Conclusion.}

We have assumed that the fr acture proceeds in the self similar manner statistically. Based on this assumption we have estimated the surface roughness associated with the microvoid growth and compared with the experiment $/ 1 \%$. Qualitative agreement betw een our analysis and the experimental observation shown in Eq. (1) demonstrates the importance of the parameters $\alpha, \beta$ and $D$ which could be determined by the experiment.

We have also postulated the initiation condition of the crack grow th which deter mines the effective surface energy $2 \gamma(\mathrm{t})$ so that the energy balance equation also holds. Making use of this postulate we could explain the experimental observation of $\mathrm{K}_{\mathrm{IC}} \propto \mathrm{t}^{-2}$. Extending this postulate to the propagation stage and evaluating the char acteristic time $t$ and time derivative of $\mathrm{K}_{\mathrm{l}}(\mathrm{t})$ from Fig. 4, we could explain the dynamic behavior of the crack velocity when it encounters with the stress wave.

\section{References}

11/ Ravi-Chander, K. and and Knauss, W.G. Inter national J. of Fracture 25 (1984) 247,26 (1984) 65, 141, 189

12/ Yokobori,T. and Narisawa, I. "The strength of the polymer materials", $\widehat{O}$ mu-sha 1982 (in Japanese)

13/ Z hurkov, S.N. and Kukshenko, V.S., Inter national J. of Fr acture Mechanics 11 (1975) 629

14/ See for example, Fur ukawa, H., Advances in Physics 34 (1985) 703

15/ Ravi-Chandar, K. and Knauss, W.G., Trans. of ASME 54 (1987) 72 